\title{
Defining New Parameters for Green Engineering Design of Treatment Reactors
}

\author{
Susana P. Boeykens ${ }^{* 1}$, Néstor Caracciolo², Cristina Vázquez ${ }^{3}$ \\ ${ }^{1}$ Laboratorio de Química de Sistemas Heterogéneos, Facultad de Ingeniería, Universidad de Buenos Aires, \\ P. Colon 850 BA, Buenos Aires, Argentina \\ e-mail: sboeyke@gmail.com \\ ${ }^{2}$ Laboratorio de Química de Sistemas Heterogéneos, Facultad de Ingeniería, Universidad de Buenos Aires, \\ P. Colon 850 BA, Buenos Aires, Argentina \\ e-mail: nestorcaracciolo@ hotmail.com \\ ${ }^{3}$ Laboratorio de Química de Sistemas Heterogéneos, Facultad de Ingeniería, Universidad de Buenos Aires, \\ P. Colon 850 BA, Buenos Aires, Argentina \\ e-mail: cvazquez@fi.uba.ar
}

\begin{abstract}
This study proposes a green way to design Plug Flow Reactors (PFR) that use biodegradable polymer solutions, capable of contaminant retaining, for industrial wastewater treatment. Usually, to the design of a reactor, the reaction rate is determined by tests on a Continuous Stirred-Tank (CST), these generate toxic effluents and also increase the cost of the design. In this work, empirical expressions (called "slip functions"), in terms of the average concentration of the contaminant, were developed through the study of the transport behaviour of $\mathrm{Cr}^{\mathrm{VI}}$ into solutions of xanthan gum. "In situ" $\mathrm{XR} \mu \mathrm{F}$ was selected as a no-invasive micro-technique to determine local concentrations. Slip functions were used with laboratory experiments planned in similar conditions using Plug Flow Reactors, to obtain useful dimensionless parameters for the industrial design.
\end{abstract}

\section{KEYWORDS}

Wastewater treatments, Biodegradable polymers, Heavy metal contaminants, Polymer retention.

\section{INTRODUCTION}

There is a wide variety of substances that pollute the environment, including metals that have achieved high levels of concentration in many areas, creating a toxic effect on living organisms [1]. In recent years, a new scientific field was developed to reduce the cost of removal treatments of metals in solution: biosorption [2]. Early works showed that some biological materials can extract metal contaminants from water at very low cost, taking advantage of their selective physicochemical properties. Additional benefits of these treatments are its public acceptance and the possibility to be used with high performance under optimal and controlled conditions. New technologies for the recovery of these metals are in development [3].

Among the biopolymers with capture capability that can be used as biomass, the Exo-Polysaccharides (EPS) are biodegradable and with an interesting low-cost production [4]. There are a great number of microbial EPS that can interact with metal

\footnotetext{
* Corresponding author
} 
ions $[5,6]$. These substances have a complex superficial chemistry with various functional groups, which together show different trends to join with other substances or ions $[7,8]$.

The classic characterization techniques for these systems are complex, laborious, with very high detection limits and low sensitivity. In addition, a considerable amount of sample to be analyzed is required. This has been a limitation to realize fast and precise experiences of transport in both, static and dynamic conditions. X-ray Micro-Fluorescence $(\mathrm{XR} \mu \mathrm{F})$ is an energy dispersive spectrometric technique with high resolution properties that allows studying dynamic systems. "In situ" $\mathrm{XR} \mu \mathrm{F}$ was selected as the no-invasive technique to determine local concentrations in this study $[9,10]$.

Usually, to the design of a Plug Flow Reactor (PFR), the reaction rate is determined by tests on a Continuous Stirred-Tank Reactor (CSTR), these generate toxic effluents and also increase the cost of the design. In this work, an empirical expression (called "slip function") in terms of the average concentration of the contaminant, was developed through the study of the transport behavior of $\mathrm{Cr}^{\mathrm{VI}}$ into solutions of xanthan gum, as a model system. Chromium, one of the most common heavy metals contaminants [11] and, xanthan, a biodegradable and low-cost EPS, were chosen for this study [12].

The presentation of a more ecological way to design PFR for treatment of aqueous industrial effluents, and the proposal of the use of biodegradable and recyclable polymers of low cost, capable of retaining contaminants is the purpose of this work.

Experimental slip functions were used with laboratory PFR experiments planned in similar conditions, to obtain useful dimensionless parameters for the industrial design.

At the present time, the study of green chemical solutions for turnover times and subsequent recycling or reuse of both the polymeric material and the recovered metal are undertaken.

\section{MATERIALS AND METHODS}

\section{Materials}

Standard Tritrisol Merck $\left(1,000 \mathrm{mgL}^{-1}\right)$ solutions of $\mathrm{Cr}$, and the same of Co (as internal standard) were used for the chromium quantification in $\mathrm{SR} \mu \mathrm{XRF} . \mathrm{K}_{2} \mathrm{CrO}_{4}$ was provided by Mallinckrodt.

Xanthan gum, provided by Sanofi Bio Industries (France), is produced by Xanthomonas campestris NRRL B-1459. It's primary structure consists of regular repeating units; each unit contains five sugars: two glucose units, two mannose units, and one glucuronic acid unit. The main chain of xanthan gum is built up of $\beta$-D-glucose units linked through the 1- and 4-positions. A three-sugar side chain is linked to the 3-position of every other glucose residue in the main chain (Figure 1).

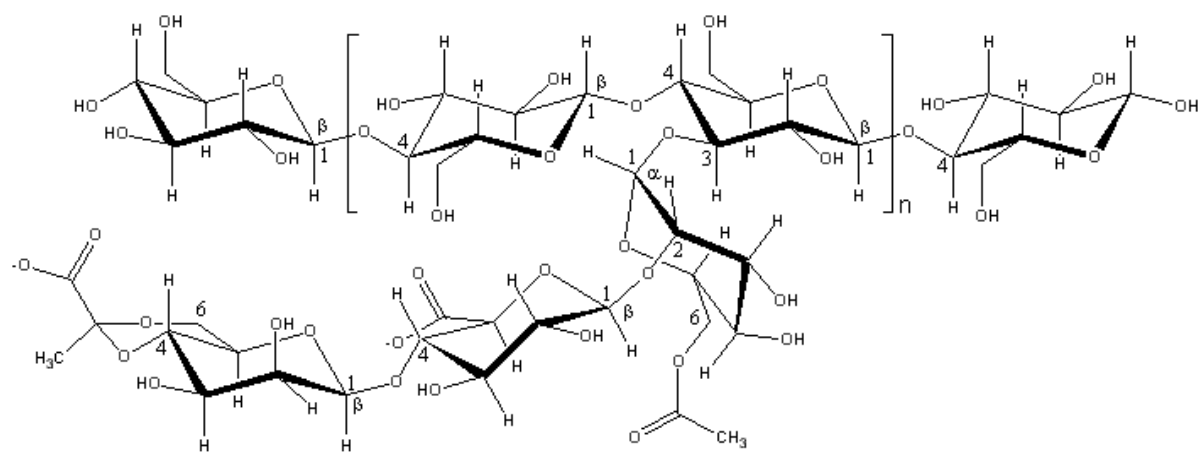

Figure 1. Xanthan gum monomer unit 
About half of the terminal D-mannose residues contain a pyruvic acid residue linked to the 4- and 6-positions. The non-terminal D-mannose unit in the side chain contains an acetyl group at position 6 . Xanthan gum is a powder that dissolves in either hot or cold water to produce solutions with high viscosity at low concentration [13, 14].

To obtain the homogeneous solutions of this polymer, a measured mass of the solid was vigorously shaken during $24 \mathrm{~h}$ in high-purity water (18 $\mathrm{M} \Omega \mathrm{cm}$ electrical resistivity). The polymeric system was purified by micro filtration and dialysis following a protocol especially tested and described in previous works [15]. The membranes used for dialysis were supplied by AH Thomas Co., (No. 4465-A2).

\section{Instrumentation}

X-ray microfluorescence. SR $\mu$ XRF was performed at the D09B-XRF line in the Laboratorio Nacional de Luz Sincrotron, Brazil (LNLS). The nominal operation energy of the storage ring is $1.37 \mathrm{GeV}$ and the maximum electron beam current is $250 \mathrm{~mA}$, with a critical photon energy of $2.08 \mathrm{keV}$. A Be $(2 \times 1 \mathrm{~mm})$ window and a Si (Li) detector with resolution energy of $140 \mathrm{eV}$ for $5.9 \mathrm{keV}$ (Mn-Ka line) were used. The highest photon flux intensity of the white beam mode was chosen. The size of the beam was $200 \times 200 \mu \mathrm{m}$.

The intensity $I_{i}$, in arbitrary units (a.u.), detected by the device is directly proportional to the concentration $C\left(\mathrm{molL}^{-1}\right)$ of the $\mathrm{Cr}$ (undifferentiating species) by a factor called sensitivity $S$ of the measurement $\left(\mathrm{Lmol}^{-1}\right)$. It was verified that the presence of any xanthan concentration does not influence the sensitivity (matrix effect) of the measurements. The utilization of the internal standard calibration technique removes any instrumental effect on the measurements. From the calibration curve using cobalt as an internal standard, the relative (to cobalt) sensitivity was obtained $\left(S_{r e l}=2,760 \mathrm{Lmol}^{-1}\right)$. In all determinations the intensity ratio $I_{\mathrm{Cr}} / I_{\mathrm{Co}}$ was calculated and the chromium concentration was obtained.

The microprobe installed at the LNLS X-Ray Fluorescence beam line station uses two types of set-ups depending on the required spatial resolution of the experiment. The set-up used in this work consists of a set of precision crossed slits giving the possibility of generating micro-beams sizes between $80 \mu \mathrm{m}$ to $200 \mu \mathrm{m}$ in both vertically and horizontally directions. The fluorescence photons are collected with the detector placed at $90^{\circ}$ to the incident beam. The SR $\mu$ XRF setup was designed for repeatability and high angular resolution as well as complete translational capacity. Translation stages were mechanically conducted to provide translations to the longitudinal axis. The originality of the measurements required the design of a special sample cell to measure transport properties into this type of systems. An improved design allows the study of two systems simultaneously (Figure 2).

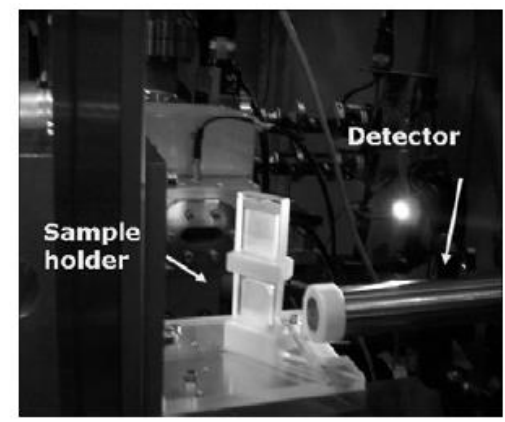

Figure 2. Special sample cells for the scanning of polymeric systems, a design improved to study simultaneously two different systems 
The experimental sample cells were designed to apply the semi-infinite free diffusion method for the determination of transport mass coefficients, similar to diffusion coefficients. In this method, the volume of solution begins at an interface and extends a length that can be considered infinite in terms of the experience: the thickness of the zone where diffusion occurs is much smaller than the total thickness of the sample. The transport of mass is a result of a pulse of concentration at the interface [16].

The main purpose of the experiences was to obtain the concentration profiles for the ions diffusing through the polymeric systems as a function of time $t$ and as a function of distance $x$ from the start point $(x=0)$.

For each experiment, $10 \mu \mathrm{L}$ of a heavy metal salt solution were deposited in the bottom container of the acrylic cell. The upper container was filled with the polymer solution. After assembling the cell $(t=0)$, it was placed in a support of the XRF line. The cell moved through the $\mathrm{x}$ axis, perpendicular to the beam, by means of a motorized stage, so that specific regions on the surface were irradiated. All measurements were performed in air-conditioned rooms $\left(25 \pm 2{ }^{\circ} \mathrm{C}\right)$.

For a complete study, samples were scanned six times requiring approximately fifteen minutes each and a set of XRF spectra with an acquisition time of $40 \mathrm{~s}$ each was obtained. Figure 3 shows a typical spectrum from xanthan solution with chromium. The total time for the diffusion into the cell was four hours and each experiment gave a collection of hundreds of spectra. The spectra were analyzed with the software package QXAS-AXIL2005, from IAEA.

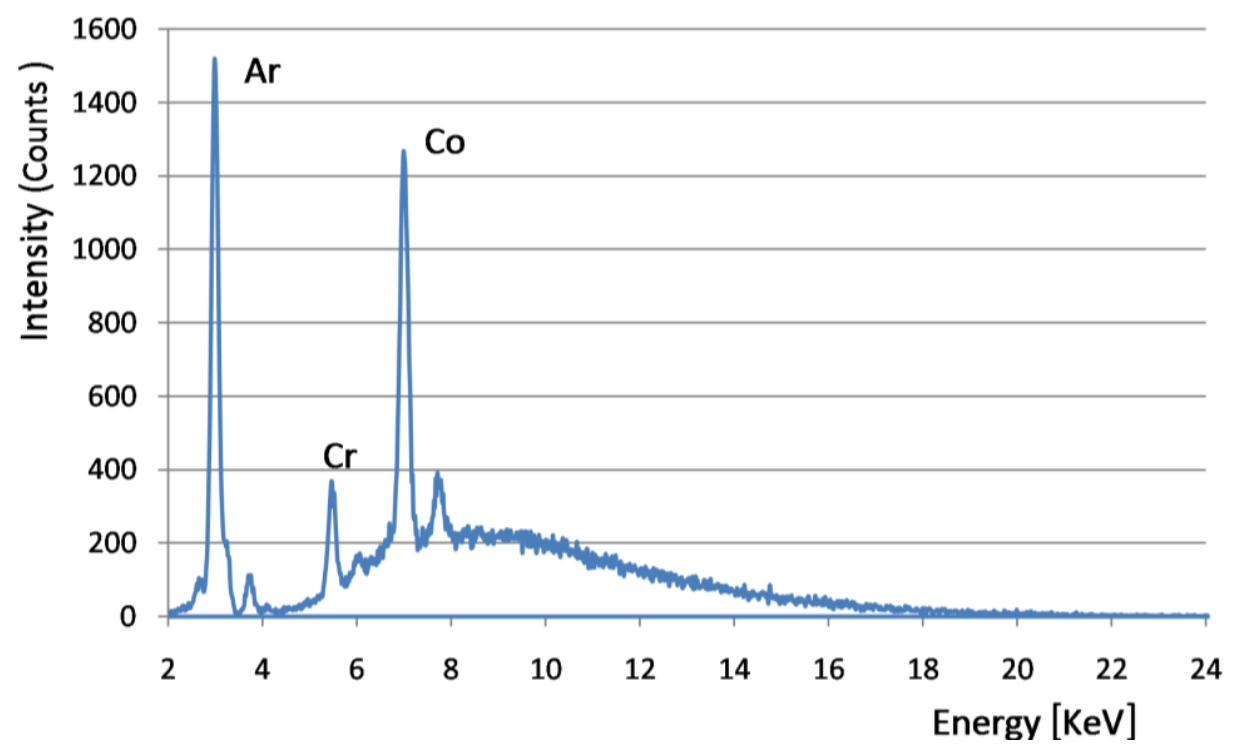

Figure 3. SR $\mu$ XRF spectrum obtained from one point in the cell with xanthan solution with chromium

Laboratory plug flow reactor. A Spectrum Labs Molecular Por cell model 76-400 was used as laboratory reactor. The fluid was driven by a peristaltic pump. The output concentration was measured with a 2400C Shimadzu UV-Vis spectrophotometer following the Standard Methods 3500-Cr B [17].

\section{RESULTS AND DISCUSSION}

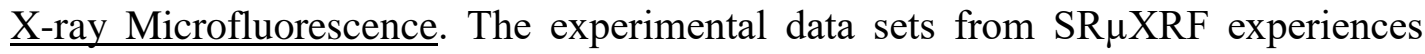
may be shown as a series of curves of concentration versus time for each position. These 
sets were obtained from the diffusion of chromium through different concentrations of xanthan gum solutions. As example, Figure 4 shows the data set corresponding to the diffusion of $\mathrm{Cr}^{\mathrm{VI}}$ through a $7,000 \mathrm{mgL}^{-1}$ xanthan gum solution.

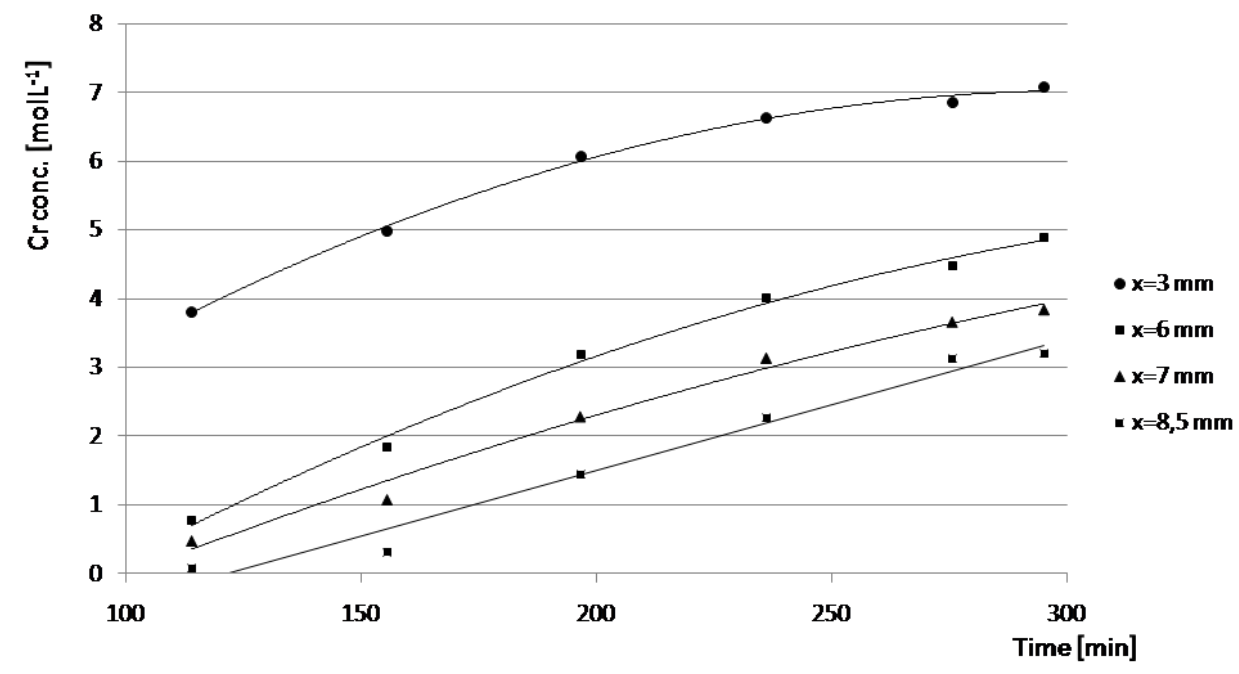

Figure 4. $\mathrm{Cr}^{\mathrm{VI}}$ concentration at different times and positions, obtained during a $\mathrm{SR} \mu \mathrm{XRF}$ scanner of the sample cell with $7,000 \mathrm{mgL}^{-1}$ xanthan solution

Slip funtions $\left(f_{s}\right)$ determination. Measuring the change in the concentration $\Delta C$ of a ion in a point $x$ at different times $t$, the increasing in mol per unit volume and time at that point could be calculated. This can be interpreted as the molar rate per unit volume at which these ions cross through that point at that time. A plot of $\Delta C / \Delta t$ as a function of the average concentrations $C_{m}$ for that interval $\Delta t$ in the same point $x$, shows the local variation of molar average rates per unit volume. Data could be fitted to a quadratic function of the type of eq. (1):

$$
f_{s}(C)=\alpha_{s} C_{m}^{2}+\beta_{s} C_{m}
$$

where $\alpha_{s}$ and $\beta_{s}$ are empirical parameters depending on the ion range of concentration and polymer concentration. The values for $\mathrm{Cr}^{\mathrm{VI}}$ in $7,000 \mathrm{mgL}^{-1}$ xantan obtained by fitting $\left(\mathrm{R}^{2}\right.$ $=0.976$ ) are shown in Table 1 .

Table 1. Experimental slip function coefficients and design dimensionless parameters obtained for the retention of $\mathrm{Cr}^{\mathrm{VI}}$ in $7,000 \mathrm{mgL}^{-1}$ xanthan solution

\begin{tabular}{ccccc}
\hline Filler solution & \multicolumn{2}{c}{ Slip function coefficients } & \multicolumn{2}{c}{ Dimensionless parameters } \\
\hline Xanthan $\left[\mathrm{mgL}^{-1}\right]$ & $\alpha_{s}\left[\mathrm{Lmol}^{-1} \mathrm{~min}^{-1}\right]$ & $\beta_{s}\left[\mathrm{~min}^{-1}\right]$ & $\theta_{s}$ & $k_{s}$ \\
\hline 7,000 & $-2.82 \times 10^{-3}$ & $1.08 \times 10^{-2}$ & $9.20 \times 10^{-1}$ & $2.79 \times 10^{-1}$ \\
\hline
\end{tabular}

Obtained by this way, the called slip function $f_{s}$ is related with the accumulation rate of the ion in this system (Figure 5). $f_{s}$ necessarily vary with the nature of the ion, the type and concentration of the polymer and the temperature. Conceptually, $f_{s}$ is a measure of the ability of the ion to stay or be retained in that system.

Higher ion rates are in dilute systems and lower in concentrated systems. However an anomaly was observed: The $f_{s}$ for xanthan $7,000 \mathrm{mgL}^{-1}$ falls below that of $8,000 \mathrm{mgL}^{-1}$. This can be explained in terms of the number of available active sites, due to easier access 
within a less concentrated solution. That is, despite there being a greater number of active sites as there are more macromolecules, not all of these sites are available because this high concentration impedes access. And then at $10,000 \mathrm{mgL}^{-1}$, a gel is formed that retains ions also by steric effects. The objective of this work is to use solutions rather than gels, by the advantages offered as its recyclability and the lower operating costs of the equipment involved (eg. lower pressure of work). The optimal range for our design will then be about 7,000 $\mathrm{mgL}^{-1}$ of xanthan, that retains the advantages of being a solution and presents a maximum of active sites.

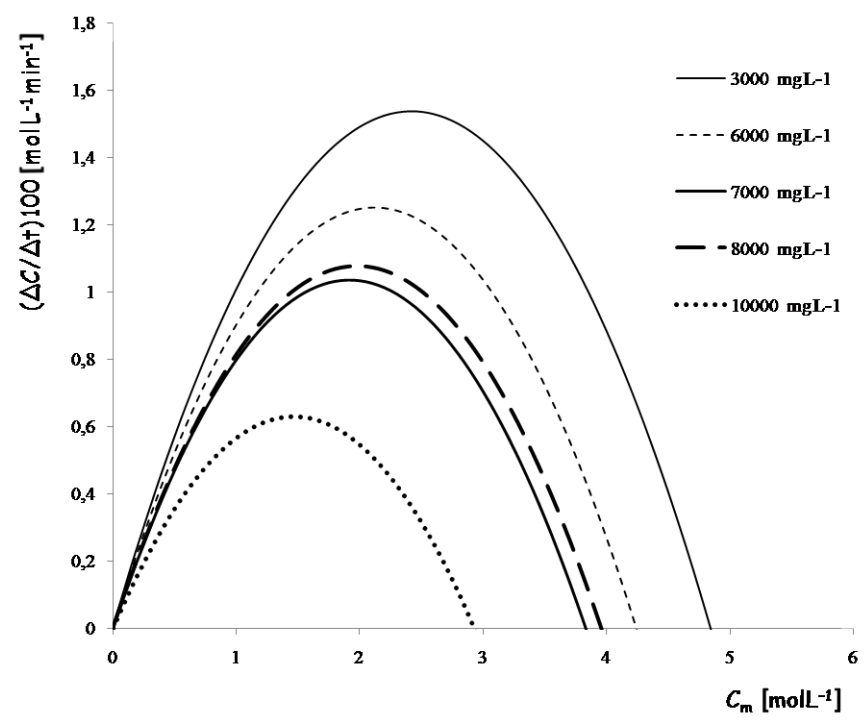

Figure 5. Slip functions obtained from chromium moving in xanthan solutions calculated from experimental data

Experiences in the PFR. In a plug flow reactor at steady state, the ion concentration in the fluid $C$ varies with the position in the direction of flow $F$, due to retention. The mass balance (eq. 2) refers to a differential volume element $d V$ moving along the reactor with a given retention rate $v_{R}$ :

$$
F d C=-v_{R} d V
$$

In this work the experimental slip functions are employed as the retention rate functions as is shown in eq. (3):

$$
v_{R}=\frac{d C}{d t}=f_{S}(C)
$$

The ion residence time $\tau_{s}$ using the slip functions, in a reactor of volume $V$ was calculated as in shown in eq. (4):

$$
\tau_{s}=\frac{\int_{0}^{V} d V}{F}=-\int_{C_{0}}^{C_{v}} \frac{d C}{f_{s}}
$$

Using the ratio of the experimental replacement time $t_{r e}$ and the residence time, fixed in this experience at 60 min (Figure 6), a dimensionless parameter, $\theta_{s}$, is generated (eq. 5). Experimental value is shown in Table 1. 


$$
\theta_{s}=\frac{t_{r e}}{60 \min }
$$

To confirm the independence of $\theta_{s}$ of the conditions of this particular experience, several similar experiments were conducted with different residence times. It was noted that $\theta_{s}$ value remains constant by doubling or by taking half the value of the volumetric flow through the reactor. Thus, a design parameter was obtained that indicates the number of residence times that any reactor may comply before that its filler should be replaced, for the given operating conditions (range of $\mathrm{Cr}^{\mathrm{VI}}$ concentrations, xanthan concentration and temperature).

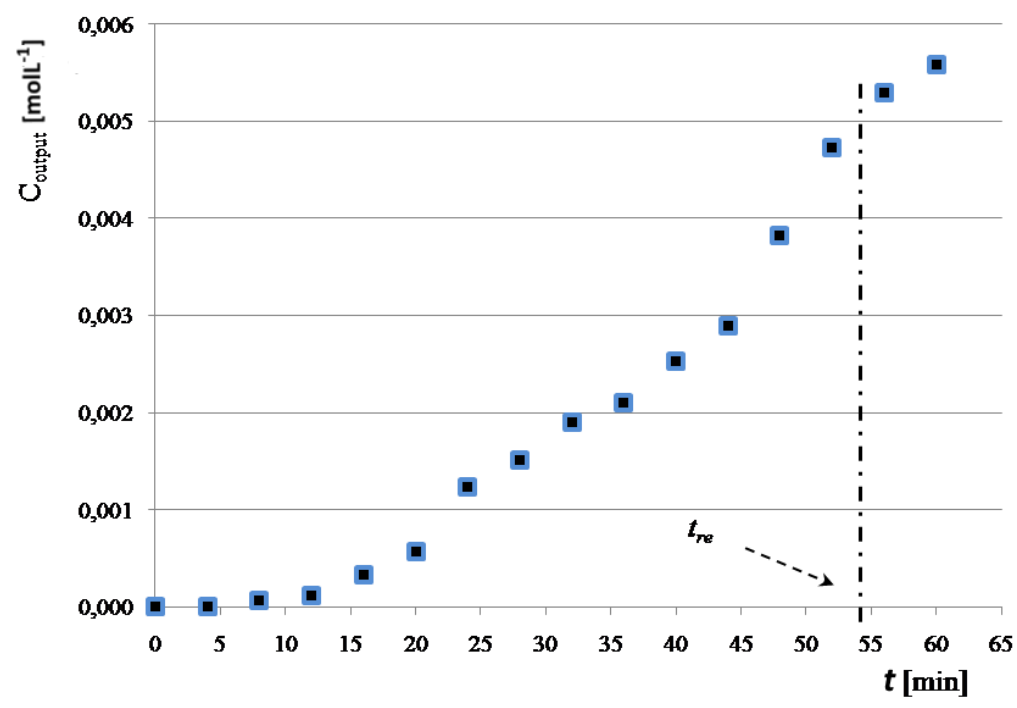

Figure 6. Output Cr concentration from the laboratory PFR reactor as a function of the time

To make the change of scale to the industrial reactor, other dimensionless parameter was generated as the ratio of the replacement time value and the value of the residence time calculated (eq. 6). Experimental value is shown in Table 1.

$$
k_{s}=\frac{t_{r e}}{\tau_{s}}
$$

The values found for this dimensionless number in experiences with different systems indicate that this is characteristic of the nature of the polymer, but does not depend on its concentration neither of the nature or concentration of the ion.

This parameter is used in the design as follows: $\tau_{s}$ is calculated by using the slip function integrated between the concentrations of interest and with $k_{s}$, the replacement time is obtained. With this value, the $\theta_{s}$ parameter is then used to obtain the residence time of the industrial reactor and to calculate its volume for a known flow. The volume thus determined maximizes the design with regard to the efficiency of the filler that was tested in a microcell, with only a few milliliters of contaminant solution.

\section{CONCLUSION}

It has been shown that the slip functions can be used as retention rate equation in reactor design. The defined dimensionless parameters $\left(\Theta_{s}, k_{s}\right)$ link between experimental values obtained in a laboratory reactor $t_{r e}$, and the values calculated using the slip 
functions $\tau_{s}$. It has been demonstrated from these experiments that the obtained values of $\theta_{s}$ can be used to estimate the time of replacement of the industrial prototype.

The presented methodology involves fewer quantities of drugs, and is faster than classical. Experiments with the laboratory PFR were essential to obtain the parameters required for the design, but, as with any engineering problem, the application will encounter compromise situations that should be resolved, involving costs and operation.

Heavy metals and the biopolymer can be recycled. When the polymer is out of stock, it can be easily cleaned and eliminated by biodegradation in digesters, thus closing the cycle of providing energy and fertilizer use.

This design method, which was described for the retention of contaminants by polymer solutions, could be applied to any other type of retention media.

In the near future when the constraints imposed by the environment were intensified and enforced, surely there will be more than one application for this kind of environmentally friendly industrial processes [18-20].

\section{AKNOWLEDGEMENTS}

This work was financially supported by UBACyT under project 20020120100201, in Argentina, and by the Brazilian Synchrotron Light Laboratory (LNLS) under proposal D09B - XRF 6514 in Brazil.

\section{NOMENCLATURE}

C Concentration

$C_{0} \quad$ Input concentration

$C_{v} \quad$ Output concentration

$C_{m} \quad$ Average concentration

$F \quad$ Flow

$T \quad$ Time

$V \quad$ Volume

\section{Greek letters}

$\alpha_{s} \quad$ coefficient of the quadratic term of the slip function

$\beta_{s} \quad$ coefficient of the lineal term of the slip function

$f_{s} \quad$ slip function

$k_{s} \quad$ dimensionless design parameter

$\theta_{\mathrm{s}} \quad$ dimensionless design parameter

$\tau \quad$ residence time

$t_{r e} \quad$ filler replacement time

$v_{R} \quad$ retention rate
$\left[\mathrm{molL}^{-1}\right]$

$\left[\mathrm{molL}^{-1}\right]$

$\left[\mathrm{molL}^{-1}\right]$

$\left[\mathrm{molL}^{-1}\right]$

$\left[\mathrm{Ls}^{-1}\right]$

[min]

[L]

\section{REFERENCES}

1. Mudhoo, A., Garg, V. and Wang, S., Heavy Metals: Toxicity and Removal by Biosorption, in: Environmental Chemistry for a Sustainable World, Vol 2., Lichfouse, E., Schwarzbauer, J., Didier, R. Eds., Springer, 2012, http://dx.doi.org/10.1007/978-94-007-2439-6_10

2. Das, N., Vimala, R. and Kartrika, P., Biosorption of Heavy Metals - An Overview, Indian Journal of Biotechnology, Vol. 7, pp. 159-169, 2008.

3. Naja, G. M., Volesky, B. and Murphy, V., Biosorption, Metals, in: Encyclopedia of Industrial Biotechnology, Flickinger, M., Wiley Interscience, NY, 2010, http://dx.doi.org/10.1002/9780470054581.eib166 
4. Kaplan, D., Christiaen, D. and Arad S., Chelating Properties of Extracellular Polysaccharides from Chlorella spp., Appl. Environ. Microb., Vol. 53, pp. 2953-2956, 1987.

5. Jeanes, A., Applications of Extracellular Microbial Polysaccharide-polyelectrolytes: Review of Literature, Including Patents, J. Polym. Sci. Polym. Symp., Vol. 45, pp 209-227, 1974, http://dx.doi.org/10.1002/polc.5070450118

6. Boeykens, S., Estudio del transporte de masa en sistemas poliméricos blandos (Mass Transport Studies in Soft Polymeric Systems), PhD Thesis, Universidad de Buenos Aires, Argentina, 2003.

7. Geesey, G. and Jang, L., Interactions between Metal Ions and Capsular Polymers, pp 325-357, in: Metal Ions and Bacteria, Beveridge, T. J, Doyle, R. J., eds., Wiley Interscience, New York, 1989.

8. Chen, J. H., Czajka, D. R., Lion, L. W., Shuler, M. L. and Ghiorse, W. C., Trace Metal in Soil by Bacterial Polymers, Environ. Health Perp., Vol. 103, pp. 53-58, 1995, http://dx.doi.org/10.1289/ehp.95103s153

9. Caracciolo, N., Boeykens, S., Vázquez, C. and Pérez, C., Ionic Transport into Soft Polymeric Systems by Micro X Ray Fluorescence, in LNLS Activity Report 2007, Westfahl, H. Jr. ed., Brazilian Association for Synchrotron Light Technology, Campinas, Brazil, 2008.

10.Boeykens, S. and Vázquez, C., Preliminary Classification of Polymers by using Total-Reflection X-ray Fluorescence Spectra, Spectrochim. Acta B, Vol. 59, No. 8, pp 1189-1192, 2004, http://dx.doi.org/10.1016/j.sab.2004.04.012

11.Dayan, A. D. and Paine, A. J., Mechanisms of Chromium Toxicity, Carcinogenicity and Allergenicity: Review of the Literature from 1985 to 2000, Human \& Experimental Toxicology, Vol. 20, No. 9, pp 439-51, 2001, http://dx.doi.org/10.1191/096032701682693062

12.Cadmus, M., Jackson, L., Burton, K., Plattner, R. and Slodki, M., Biodegradation of Xanthan Gum by Bacillus sp., Appl. Environ. Microbiol., Vol. 44, No. 1, pp 5-11, 1982.

13.Jansson, P., Kenne, L. and Lindberg, B., Structure of the Extracellular Polysaccharide from Xanthomonas Campestris, Carbohydr. Res., Vol. 45, pp 275-282, 1975, http://dx.doi.org/10.1016/S0008-6215(00)85885-1

14.Jeanes, A., Rogovin, P., Cadmus, M. C., Silman, R. W. and Knutson, C. A., Polysaccharide (Xanthan) of Xanthomonas Campestris NRRL B-1459: Procedures for Culture Maintenance and Polysaccharide Production, Purification, and Analysis, ARS-NC-51, pp 1-14. U.S., Agricultural Research Service, North Central Region, U.S. Department of Agriculture, Washington, D.C., 1976.

15.Boeykens, S., Temprano, N. and Vázquez, C., Low Impurities in Scleroglucan Systems: Removal Processes Control by Total Reflection X-ray Fluorescence, J. Trace Microprobe Techn., Vol. 20, pp 283-292, 2002, http://dx.doi.org/10.1081/TMA-120003730

16.Cussler, E. L., Diffusion: Mass Transfer in Fluid Systems, $3^{\text {rd }}$ Ed., Cambridge University Press, NY, USA, 2009, http://dx.doi.org/10.1017/СВ09780511805134

17.APHA, AWWA, WEF, Standard Methods for the Examination of Water \& Wastewater, $22^{\text {nd }}$ Ed., 3500-Cr B. Colorimetric Method, 2012.

18.Poder Ejecutivo Nacional, Decreto 831/93, Residuos peligrosos, Reglamentación de la Ley 24051 (Hazardous waste, Regulation of Law 24051), Boletín Oficial de la República Argentina, N 27630 (3/5/93), pp 4-22, 1993.

19.Sodhi, V. and Sohpal, V., Treatment of Chromium (VI) Containing Aqueous Effluent of Tanneries and Electroplating Units by Membrane Process, 227-1, Proceedings of the International Conference on Environmental Pollution and Remediatio, Ottawa, Ontario, Canada, 17-19 August 2011, pp 227-1 - 227-8, 2011. 
20.Mendez Pampin, R., Vidal Saenz, G., Lorber, K. and Romegialli, F., Producción limpia en la industria de la curtiembre (Cleaner production in tanning industry), Universidad de Santiago de Compostela, España, 2009.

Paper submitted: 21.08 .2015

Paper revised: 06.10.2015

Paper accepted: 11.10 .2015 